\section{Anatomical success rate of macular hole surgery with autologous platelet without internal- limiting membrane peeling}

CMG Cheung, V Munshi, S Mughal, J Mann and $\mathrm{M}$ Hero

\begin{abstract}
Aim To describe our experience and success rate of macular hole surgery with pars plana vitrectomy with autologous platelet and without internal limiting membrane peel. Methods Retrospective review of 56 consecutive patients who underwent macular hole surgery.

Results Anatomical success was achieved in 55 out of 56 patients (98.2\%). Functional success was achieved in 37 out of 56 patients (66.1\%). A total of 21 patients (37.5\%) achieved postoperative visual acuity of $6 / 12$ or better. No intraoperative complications were encountered. Postoperative complications included cataract progression in eight eyes and raised intraocular pressure in 20 eyes.

Conclusions Our success rate was comparable to that reported in macular hole surgery incorporating internal limiting membrane (ILM) peel or with autologous platelet without ILM peel.

Eye (2005) 19, 1191-1193. doi:10.1038/sj.eye.6701733; published online 18 March 2005
\end{abstract}

Keywords: macular hole surgery; autologous platelets; internal limiting membrane peel

\section{Introduction}

It has been postulated that contractile forces generated by glial cells migrating onto the inner surface of internal limiting membrane (ILM) play a significant role in the pathogenesis of idiopathic macular holes. ${ }^{1}$ The combination of ILM peel with pars plana vitrectomy (PPV) in the treatment of macular holes has been shown to achieve high success rate (91-100\% anatomically and $85 \%$ functionally). ${ }^{2-5}$ However, ILM peel may increase the risk of retinal breaks, retinal haemorrhage, and postoperative epiretinal membrane formation. ${ }^{2}$ Furthermore, incomplete or unsuccessful ILM peeling may induce a stronger stimulation of gliosis, which is associated with worse visual outcome. ${ }^{6}$ Uemoto $e t ~ a l^{7}$ have also shown that while optical coherence tomography (OCT) anatomical closure rate is higher after ILM peel, no significant difference was found in the clinical visual improvement. On the other hand, a high rate of macular hole closure (93.9-98\%) has also been reported by using autologous platelet concentrate as adjuvant without ILM peel..$^{8-12}$ In this study, we report the success rate of macular hole surgery with autologous platelet, without ILM peel.

\section{Patients and methods}

A total of 56 consecutive patients who underwent macula hole surgery with visual acuity of $6 / 12$ or worse were included. Patients with macular hole diameter of over $1000 \mu \mathrm{m}$ were excluded. The length of visual symptom was not used in the selection criteria for surgery. Data collected included age, sex, stage, and size of macular hole, best-corrected preoperative visual acuity, lens status, tamponade agent, intraoperative and postoperative complications, anatomical outcome, and best-corrected postoperative visual acuity. Anatomical success is defined as complete disappearance of subretinal fluid and flattening of the entire circumference of the macular hole. Functional success is defined as improvement of two or more lines of Snellen acuity.
University Hospitals Coventry and Warwickshire NHS Trust, Birmingham, UK

Correspondence: CMG Cheung, Specialist Registrar, Eye Department, Birmingham \& Midland Eye Centre, City Hospital, Dudley Road, Birmingham B18 7QU, UK

Tel: + 447990807649 ;

Fax: +44121247 1434 .

E-mail: gemmycheung@ doctors.org.uk

Received: 29 April 2004 Accepted in revised form: 2 September 2004 Published online: 18 March 2005 


\section{Surgery}

All cases were carried out under general anaesthetics by a single vitreoretinal surgeon at Coventry and Warwick Hospital. The macular hole surgery included induction of posterior vitreous detachment, PPV, fluid-air exchange, injection of autologous platelets, and injection of $20 \%$ sulphur hexafluoride $\left(\mathrm{SF}_{6}\right)$ or $18 \%$ perfluoropropane $\left(\mathrm{C}_{3} \mathrm{~F}_{8}\right)$ gas. Where significant cataract was present preoperatively, simultaneous phacoemulsification with intraocular lens implantation was also performed in 20 patients. All patients were instructed to posture face-down until the gas bubble disappeared.

\section{Results}

Out of the 56 patients, 39 were female and 17 were male. The age ranged from 58 to 79 years. The preoperative visual acuity ranged from $6 / 12$ to below $6 / 60$, with $6 / 36$ or worse in 42 patients (75\%). In all, eight eyes had stage II macular hole, 36 eyes had stage III, and 12 eyes had stage IV. The preoperative hole age was not used for inclusion criteria for surgery as this is often inaccurate. However, it was noted that 39 patients had symptoms for less than 6 months and 17 patients had symptoms for over 6 months. In total, 36 eyes underwent PPV, fluid-air exchange, injection of autologous platelets, and injection of expansile gas. A total of 20 eyes were noted to have significant cataracts preoperatively and underwent phacoemulsification of cataract and intraocular lens implantation combined with macular hole surgery as described. $\mathrm{C}_{3} \mathrm{~F}_{8}$ was used in 40 eyes whereas $\mathrm{SF}_{6}$ was used in 16. There were no intraoperative complications. Postoperatively raised intraocular pressure (over $25 \mathrm{mmHg}$ ) was noted in 20 eyes. Cataract progression was noted in eight eyes.

Anatomical success with one operation was achieved in 55 out of 56 patients (98.2\%). One patient was found to have incomplete gas fill on the first postoperative day and underwent reinsertion of gas and platelets.

Functional success was achieved in 37 out of 56 patients $(66.1 \%)$. The length of follow-up was less than 6 months in 37 patients, up to 12 months in 16 patients, up to 18 months in one patient, and over 18 months in two patients. The final visual acuity ranged from $6 / 9$ to below 6/60. Overall, 21 (37.5\%) patients achieved visual acuity of $6 / 12$ or better postoperatively.

\section{Discussion}

The effect of autologous platelet as an adjunct in surgery for macular hole repair has been studied in a multicentre, prospective, double-masked randomized controlled trial involving 110 eyes. ${ }^{9}$ Vitrectomy with autologous platelet concentrate (APC) achieved anatomical success rate of $98 \%$ compared with $82 \%$ with vitrectomy alone.

Postoperative visual acuity improved by 9.7 ETDRS score (preop mean 50.3, postop mean 59.8) in the vitrectomy with APC group compared to 6.7 in the vitrectomy group. This was not statistically significant. No complications from endophthalmitis was reported.

Vitrectomy with ILM peeling has been reported to achieve high anatomic (100\%) and visual success rate in Brooks' series. ${ }^{3}$ This is a retrospective, nonrandomized comparative trial. High success for chronic macular holes (anatomic closure 97\% for holes greater than 6 months), reopened and failed macular holes $(100 \%)$ was also reported. However, it is worth noting that this series contains many stage II holes $(44.8 \%)$ and smaller holes (all less than $500 \mu \mathrm{m}$ ). Even the chronic holes are all less than $600 \mu \mathrm{m}$. These factors may influence the final vision. Furthermore, another study has found no significant difference in visual acuity between groups with and without ILM peeling. ${ }^{12}$ Brooks also found that stage II holes may close without ILM peeling, with comparable visual acuities to those closed with ILM peeling.

The proposed role of ILM peel include release of tangential traction of prefoveal vitreous or epiretinal cellular constituents and glial cell proliferation and contraction causing centripetal movement of photoreceptors. ${ }^{8}$ Consequently, ILM peeling has been associated with higher anatomical success rate. However, this has not always been translated into higher functional success. The use of autologous platelet concentrate as an adjuvant to vitrectomy has also been shown to offer high success rate without the need for ILM peel. Surgery without ILM peeling reduces the risk of inducing retinal breaks, retinal haemorrhages, and postoperative epiretinal membrane formation. Potential disadvantages of autologous platelets include the need for special handling, added expense, and the risk of

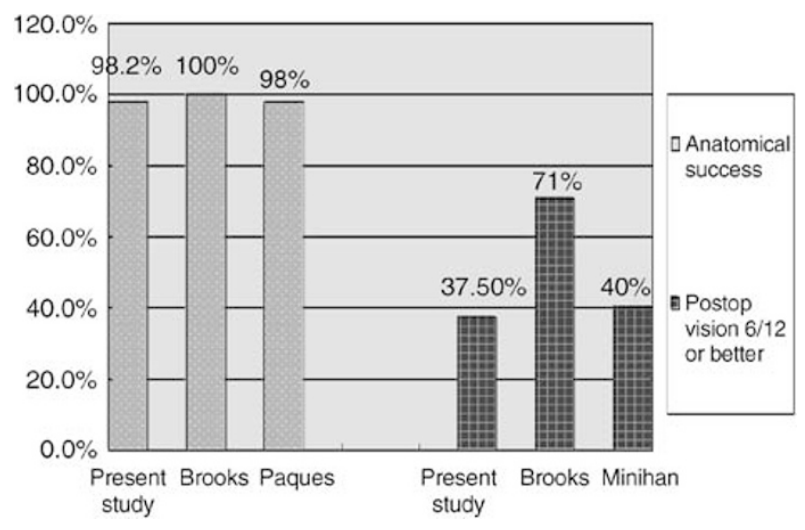

Figure 1 Anatomical success rate and visual outcome: comparison of our results with published studies. 
endophthalmitis. Our success rate was comparable to that reported in macular hole surgery incorporating ILM peel $^{2-5}$ or with autologous platelet without ILM peel ${ }^{9-12}$ (Figure 1).

Anatomical success has not always translated into functional success in this and various previous studies. Visual improvement depends on many more variables including hole stage, hole size, hole age, and the status of the lens. We conclude that vitrectomy with autologous platelets but without ILM peel is safe and provides comparable success rate to surgery with ILM peel.

\section{References}

1 Yoon H, Brooks HL, Capone Jr A, L'Hernault NL, Grossniklaus HE. Ultrastructural features of tissue removed during idiopathic macular hole surgery. Am J Ophthalmol 1996; 122: 67-75.

2 Park DW, Sipperley JO, Sneed SR, Dugel PU, Jacobsen J. Macular hole surgery with internal-limiting membrane peeling and intravitreous air. Ophthalmology 1999; 106: 13921397.

3 Brooks HL. Macular hole surgery with and without internal limiting membrane peeling. Ophthalmology 2000; 107: 1939-1949.

4 Mester V, Kuhn F. Internal limiting membrane removal in the management of the full-thickness macular holes. Am J Ophthalmol 2000; 129: 769-777.

5 Al-Abdulla NA, Thampson JT, Sjaarda RN. Results of macula hole surgery with and without epiretinal dissection or internal limiting membrane removal. Ophthalmology 2004; 111: $142-149$.

6 Smiddy W, Feuer W, Cordahi G. Internal limiting membrane peeling in macular hole surgery. Ophthalmology 2001; 108: 1471-1478.

7 Uemoto R, Yamamoto S, Aoki T, Tsukahara I, Yamamoto T, Takeuchi S. Macular configuration determined by optical coherence tomography after idiopathic macular hole surgery with or without internal limiting membrane peeling. Br J Ophthalmol 2002; 86: 1240-1242.

8 Funata M, Wendel RT, de la Cruz Z, Green WR. Clinicopathologic study of bilateral macular holes treated with pars plana vitrectomy and gas tamponade. Retina 1992; 12: 289-298.

9 Paques M, Chastang C, Mathis A, Sahel J, Massin P, Dosquet $C$ et al. Effect of autologous platelet concentrate in surgery for idiopathic macular hole: results of a multicentre, double-masked, randomized trial. Platelets in macular hole surgery group. Ophthalmology 1999; 106: 932-938.

10 Minihan M, Goggin M, Cleary PE. Surgical management of macular holes: results using gas tamponade alone, or in combination with autologous platelet concentrate, or transforming growth factor beta 2. Br J Ophthalmol 1997; 81: 1073-1079.

11 Hoerauf H, Kluter H, Joachimmerg E, Roider J, Framme C, Schlenke P et al. Results of vitrectomy and the no-touchtechnique using autologous adjuvants in macular hole treatment. Int Ophthalmol 2001; 24: 151-159.

12 Olsen TW, Sternberg Jr P, Capone Jr A, Martin DF, Lim JI, Grossniklaus HE et al. Macular hole surgery using thrombin-activated fibrinogen and selective removal of the internal limiting membrane. Retina 1998; 18: 322-329. 